CASE REPORT

\title{
Sudden infant death triggered by dive reflex
}

\section{Matturri, G Ottaviani, A M Lavezzi}

J Clin Pathol 2005;58:77-80. doi: 10.1136/icp.2004.020867

The dive reflex is the reflex mechanism most frequently considered in the aetiopathogenesis of sudden infant death syndrome (SIDS). This seems to persist in human beings as an inheritance from diver birds and amphibians. It has been reported that washing the face with cold water or plunging into cold water can provoke cardiac deceleration through the intervention of the ambiguus and the vagal dorsal nuclei. This report describes a case of SIDS that offers a unique insight into the role of the dive reflex in determining a lethal outcome. Examination of the brainstem on serial sections revealed severe bilateral hypoplasia of the arcuate nucleus and gliosis of the other cardiorespiratory medullary nuclei. The coronary and cardiac conduction arteries presented early atherosclerotic lesions. The possible role of parental cigarette smoking in the pathogenesis of arcuate nucleus hypoplasia and early coronary atherosclerotic lesions is also discussed.

"From birth I was cast upon you; from my mother's womb you have been my God" (Psalm, 22: 10)

T

he sudden infant death syndrome (SIDS), or crib death, is defined as the sudden death of an infant under 1 year

of age, which remains unexplained after a thorough case investigation, including the performance of a complete necropsy, examination of the death scene, and a review of the clinical history.'

The most important pathogenic hypotheses of SIDS are: a respiratory (apnoea), a cardiac (arrhythmogenic), or a visceral dyskinetic (glottal spasm and/or oesophagogastric reflux) cause. $^{2-4}$ It has been stressed that the autonomic nervous system (respiratory, cardiovascular, and upper digestive) must interact in all such aetiopathogenic hypotheses. ${ }^{5-7}$

The dive reflex is the reflex mechanism most frequently considered in the aetiopathogenesis of SIDS. ${ }^{8}{ }^{9}$ However, few, if any, cases of infants dying after immersion in a swimming pool have been reported, and no association between parental cigarette smoking and either arcuate nucleus hypoplasia or early atherosclerosis has been proposed.

Herein, we report a case of sudden and unexpected infant death that offers a unique insight into the role of a delayed dive reflex in determining the lethal outcome, in addition to the role that parental cigarette smoking might have played in the pathogenesis of the observed arcuate nucleus hypoplasia and early coronary atherosclerotic lesions.

\section{CASE REPORT}

A 10 month old male infant presented with vomiting and dysentery during a swimming lesson, after having been immerged in cold water. He was taken to the emergency room, where a gastroenteric disturbance of possible viral

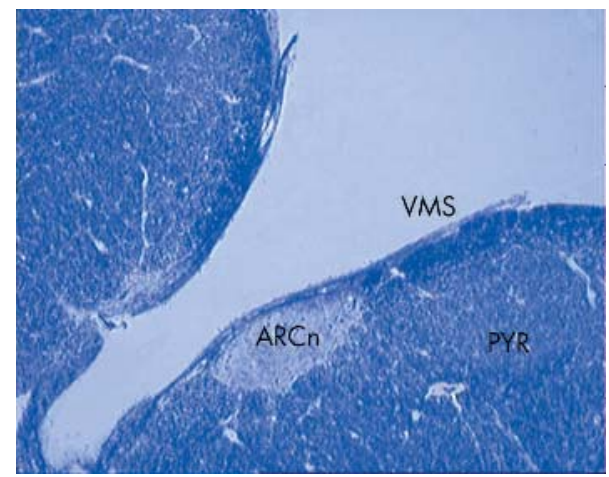

Figure 1 Bilateral hypoplasia of the arcuate nucleus (ARCn). VMS, ventral medullary surface; PYR, pyramid. Klüver-Barrera stain; original magnification, $\times 25$.

origin was diagnosed. He was rehydrated and discharged. The following night, while lying in his mother's arms, the baby suddenly became unresponsive. Emergency help was summoned. Resuscitation efforts were attempted by external cardiac massage and assisted ventilation, followed by the repeated administration of adrenaline, but the child remained unconscious and was pronounced dead a few minutes later.

The baby had been born at term, after an uncomplicated pregnancy. He seemed to be normal at birth and continued in apparent good health until the day he died. The baby was bottle fed and both parents were cigarette smokers (two packs/day).

No previous electrocardiogram recordings were available. A postmortem examination was requested for the clinical suspicion of SIDS. The case was referred to the institute of pathology, University of Milan, Italy, for the performance of specialist investigations.

\section{METHODS}

A complete necropsy was performed, including close examination of the cardiac conduction system and of the central and peripheral autonomic nervous structures involved in cardiorespiratory reflexogenesis, according to the protocol routinely followed in our institute, available online (http:// users.unimi.it/ pathol/sids_e.html). ${ }^{46-8}$

The medulla oblongata was divided into three blocks. The first (cranial) block extended from the border between the medulla oblongata and pons up to the upper pole of the olivary nucleus. The second (intermediate) block, corresponding to the submedian area of the inferior olivary nucleus, took the obex as the reference point, and extended 2-3 $\mathrm{mm}$ above and below the obex itself. The third (caudal) block included the lower pole of the inferior olivary nucleus and the lower adjacent area of the medulla oblongata. The first and second blocks were sectioned in a cranial-caudal

Abbreviations: H\&E, haematoxylin and eosin; SIDS, sudden infant death syndrome 




Figure 2 Aspects of gliosis throughout the brainstem. Haematoxylin and eosin stain; original magnification, $\times 200$.

direction, and the third in a caudal-cranial direction. ${ }^{6} \mathrm{~A}$ fourth block of the brainstem included the ponto-mesencephalic portion and was sectioned in a caudo-rostral direction. From each of these paraffin wax embedded blocks, an average of 48 sections were obtained and were alternately stained using haematoxylin and eosin (H\&E), Bielschowsky, and Klüver-Barrera stains. ${ }^{6} 10$

Morphometric analysis was performed with an Image-Pro Plus Image analyser (Media Cybernetics, Silver Spring, Maryland, USA) on both sides of the brainstem. The volume of the arcuate nucleus was measured by two dimensional reconstruction.

After the presence of gross cardiac malformations had been excluded, the origins of the coronary arteries were carefully inspected. The heart structures were routinely examined for pathological changes in the atria, septa, ventricles, pericardium, endocardium, and coronary arteries. Samples of the myocardium and the major coronary arteries (left main, left anterior descending, left circumflex, right main, right posterior descending, and right marginal) were stained with $\mathrm{H} \& \mathrm{E}$ and trichromic Heidenhain (Azan). ${ }^{11}$

The cardiac conduction system was removed in two blocks: the first included the sinoatrial node and the crista terminalis; the second contained the atrioventricular node, His bundle down to the bifurcation, and bundle branches. These two blocks were cut serially at intervals of $40 \mu \mathrm{m}$ (levels) and alternately stained with H\&E and Azan. ${ }^{41}$

\section{PATHOLOGICAL FINDINGS}

At necropsy, the baby was described as a well developed, well nourished infant, with body length and weight within the normal ranges. There was no evidence of malformations or marks of violence. The external and internal examinations were entirely normal for the age and sex, showing only subpleural petechiae over the lungs.

A histopathological examination of the brain disclosed perineural and perivascular oedema, and mild gliosis.
Examination of the brainstem on serial sections revealed severe bilateral hypoplasia of the arcuate nucleus, as confirmed by two dimensional morphometric reconstruction (fig 1). Diffuse astrogliosis of the other cardiorespiratory medullary nuclei-namely, the hypoglossus, the dorsal vagus motor, the tractus solitarii, the ambiguus, the trigeminal nuclei, the ventrolateral reticular formation, and the parabrachial/Kölliker-Fuse complex-was also noted (fig 2).

The coronary arteries presented early atherosclerotic lesions marked by infiltration in the intima of smooth muscle cells, monocytes/foam cells, rare B cells, and deposits of amorphous material mainly consisting of mucopolysaccharides, even in the innermost portion of the media, in addition to fragmentation of the elastic fibre system (fig 3).

Histological examination of the cardiac conduction system showed the sinoatrial node and its adjacent ganglia to be normal. Islands of conduction tissue, known as persistent fetal dispersion, were observed in the central fibrous body, as well as areas of resorptive degeneration ${ }^{4}$ in the atrioventricular node. Thickening of both the sinoatrial node (fig 4) and atrioventricular node arteries was noted (fig 5).

No other relevant pathological changes were found. In particular, no inflammatory or infective lesions were detected in the gastrointestinal apparatus.

\section{DISCUSSION}

Overall, the abnormalities of the autonomic nervous and cardiac conduction systems described in our case represent a plausible basis for the diagnosis of a reflexogenic SIDS of vagal inhibitory nature following a dive reflex. ${ }^{8}$

It has been noted that some newborns with a developmental defect of the brainstem reflexogenic centres, interacting through the glossopharyngeal or trigeminal nerves, die of apnoea and cessation of breathing. ${ }^{8912}$ The dive reflex seems to persist in humans as an inheritance from diver birds and amphibians. It has been reported that in humans washing the face or plunging into cold water can provoke cardiac deceleration through the intervention of the ambiguus and the vagal dorsal nuclei. ${ }^{8}$

The anomalous cardiac conduction, brainstem (figs 1, 2), and coronary (figs 3-5) morphological findings detected in our present case have already been described in infants dying suddenly and unexpectedly. ${ }^{4-6}{ }^{11}{ }^{13}$ In particular, congenital abnormalities of the medullary arcuate nucleus ${ }^{6}{ }^{13}$ and of the cardiac conduction system ${ }^{4}$ have been described as possible morphological substrates for sudden reflexogenic infant death. ${ }^{8}$ Frequent developmental abnormalities in the brainstem, particularly in the arcuate nucleus, have been identified in SIDS and sudden unexplained perinatal death. ${ }^{5-8}$ The arcuate nucleus is an important cardiorespiratory centre of the ventral medullary surface, characterised by high morphological variability. ${ }^{5}$ Arcuate nucleus hypoplasia, which is detected in over $50 \%$ of infants dying of $\operatorname{SIDS}^{14}$ and in $35 \%$ of stillborn babies, ${ }^{7}$ is of great interest, particularly because of its frequency and its pathogenic implications. Sometimes, it is

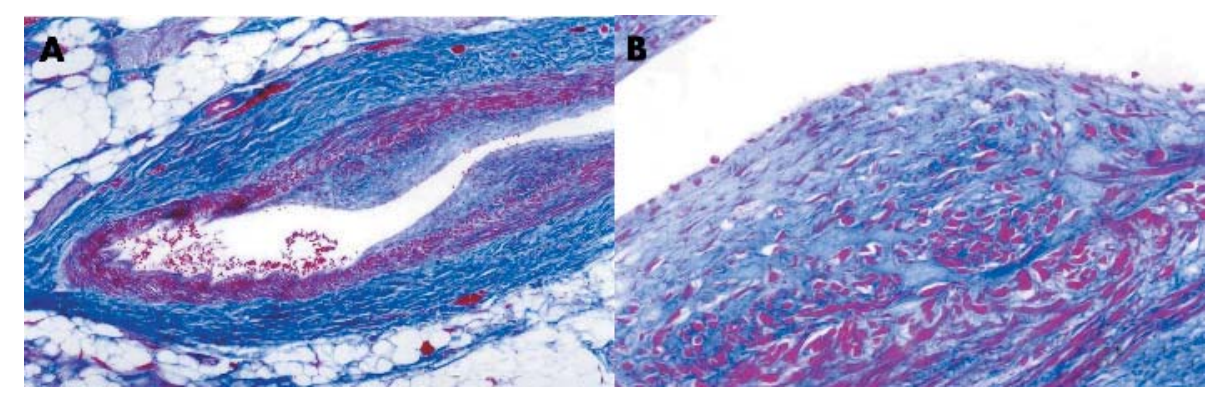

Figure 3 Histological section of the circumflex branch of the left coronary artery showing two opposed early atherosclerotic lesions, consisting of soft thickening with numerous foam cells and narrowing of the coronary lumen. Trichromic Heidenhain (Azan) stain; original magnification, (A) $\times 25$ and (B) $\times 200$. 




Figure 4 Narrowing as a result of an early atherosclerotic lesion of the sinoatrial node artery; SAN, sinoatrial node. Azan stain; original

magnification, $(A) \times 25$ and $(B) \times 400$.

associated with alterations in other brainstem structures, such as the respiratory reticular formation, and with pulmonary hypoplasia in near term fetuses. ${ }^{7}$ Mahaim fibres are reported to be statistically more frequent in infants dying of SIDS who have arcuate nucleus hypoplasia than in those infants with a well developed arcuate nucleus, whereas other cardiac conduction findings are unrelated to the arcuate hypoplasia (L Matturri L, et al. Sudden infant death syndrome (SIDS): associated alterations of the cardiac conduction system and brainstem. Proceedings of the seventh SIDS international conference. Florence, Italy: 2002:133).

"The abnormalities of the autonomic nervous and cardiac conduction systems described in our case represent a plausible basis for the diagnosis of a reflexogenic sudden infant death syndrome of vagal inhibitory nature following a dive reflex"

In our case, the unexpected death was triggered by a dive reflex as a result of bathing in the swimming pool, particularly temporarily plunging the face under water. Solicitation of the reflected neuronal circuits that participate in modulation of the respiratory, cardiovascular, and also digestive activities, ${ }^{468}$ seems to be responsible for the symptoms of regurgitation and liquid diarrhoea manifested by the baby.

Histological examination of the brainstem on serial sections revealed bilateral hypoplasia of the arcuate nucleus. Maternal smoking during pregnancy is significantly and independently associated with SIDS. ${ }^{15}$ Storm et al found an increase in brainstem reactive astrogliosis, mainly in the inferior olivary nucleus, in perinatal and SIDS victims prenatally exposed to cigarette smoke. ${ }^{16}$ We have recently shown a significantly increased incidence of cytoarchitectural alterations of the arcuate nucleus in stillborns and in SIDS victims with mothers who smoke compared with those

\section{Take home messages}

- We describe a case of reflexogenic sudden infant death syndrome (SIDS) of vagal inhibitory nature triggered by a dive reflex as a result of bathing in the swimming pool, particularly temporarily plunging the face under water

- Examination of the brainstem on serial sections revealed severe bilateral hypoplasia of the arcuate nucleus and gliosis of the other cardiorespiratory medullary nuclei; in addition, the coronary and cardiac conduction arteries presented early atherosclerotic lesions

- We suggest that parental cigarette smoking may play a role in the pathogenesis of arcuate nucleus hypoplasia and early coronary atherosclerotic lesions

whose mothers do not smoke. We found, in both stillborns and infants, a wide variety of structural defects of the arcuate nucleus, ranging from neuronal immaturity in a well shaped structure to total agenesis. ${ }^{13}$

In our study, the histological examination of serially sectioned coronary and cardiac conduction arteries revealed the presence of atherosclerotic lesions (figs 3-5). In recent studies we have reported that early atherosclerotic or preatherosclerotic lesions are already detectable in infancy, ${ }^{11}$ and even during fetal life, ${ }^{17}$ and are significantly related to parental cigarette smoking ${ }^{11}$ and formula feeding. ${ }^{11}{ }^{17}$

In this case, accurate examination of the brainstem, coronary arteries, and cardiac conduction system on serial sections was crucial to the identification of the morphological substrates responsible for this reflexogenic sudden death.

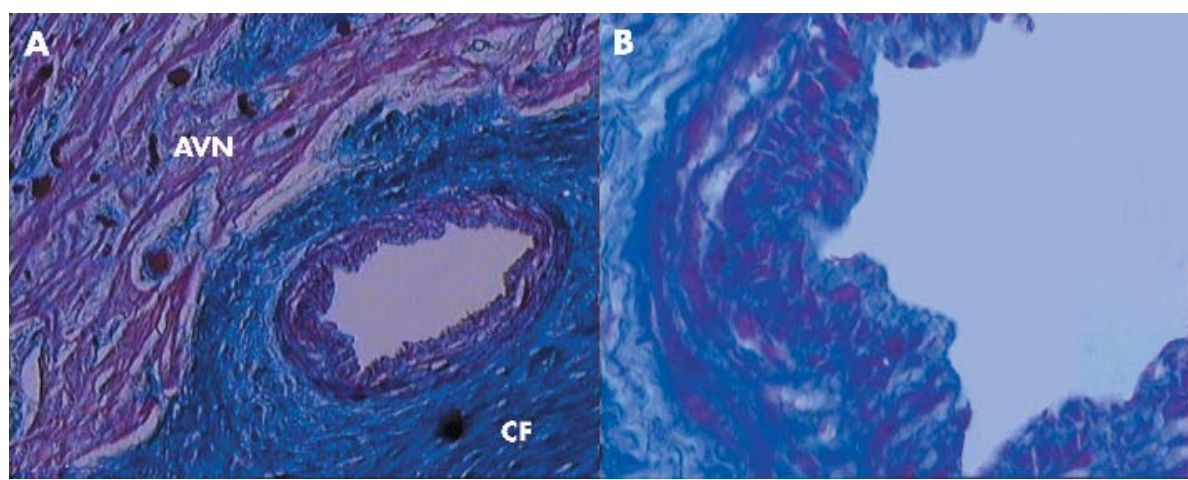

Figure 5 Narrowing as a result of an early atherosclerotic lesion of the atrioventricular node artery. AVN, atrioventricular node; CF, central fibrous body. Azan stain; original magnification, (A) $\times 25$ and $(B) \times 400$ 


\section{ACKNOWLEDGEMENTS}

We thank Mrs G Alfonsi and Mrs D Tosi for skillful technical cooperation. This study was supported by the Ministry of Foreign Affairs (joined project of particular relevance number 269/P/0085087 "Anatomopathologic and genetic study of the unexplained perinatal death and SIDS") and by "Lino Rossi" Research Centre for the study and prevention of unexpected perinatal death and sudden infant death syndrome (SIDS) funding (rectorial decree number 225678 of 23/04/04).

\section{Authors' affiliations}

L Matturri, G Ottaviani, A M Lavezzi, "Lino Rossi" Research Centre for the study and prevention of unexpected perinatal death and sudden infant death syndrome (SIDS), Institute of Pathology, University of Milan, 20122 Italy

Website: http://users.unimi.it/ pathol/pathol_e.html

Correspondence to: $\operatorname{Dr} \mathrm{G}$ Ottaviani, Institute of Pathology, University of Milan, Via della Commenda, 19, 20122 Milan, Italy; giulia.ottaviani@ unimi.it

Accepted for publication 27 July 2004

\section{REFERENCES}

1 Willinger M, James LS, Catz C. Defining the sudden infant death syndrome (SIDS): deliberations of an expert panel convened by the National Institute of Child Health Development. Pediatr Pathol 1991;11:677-84.

2 Guntheroth WG, Spiers PS. Prolongation of the QT interval and the sudden infant death syndrome. Pediatrics 1999;10:813-14.

3 Guntheroth WG, Spiers PS. Are bedding and rebreathing suffocation a cause of SIDS? Pediatr Pulmonol 1996;22:335-41.
4 Ottaviani G, Matturri L, Rossi L, et al. Crib death: further support for the concept of fatal cardiac electrical instability as the final common pathway. Int J Cardiol 2003;92:17-26.

5 Kinney HC, Filiano JJ, Harper RM. The neuropathology of the sudden infant death syndrome. A review. J Neuropathol Exp Neurol 1992;51:115-26.

6 Matturri L, Ottaviani G, Alfonsi G, et al. Study of the brainstem, particularly the arcuate nucleus, in sudden infant death syndrome (SIDS) and sudden intrauterine unexplained death (SIUD). Am J Forensic Med Pathol 2004; $25: 44-8$.

7 Matturri L, Lavezzi AM, Minoli I, et al. Association between pulmonary hypoplasia and hypoplasia of arcuate nucleus in stillbirth. J Perinatol 2003;23:328-32

8 Rossi L. Bulbo-spinal pathology in neurocardiac sudden death of adults: a prognostic approach to a neglected problem. Int J Legal Med 1999:1 12:83-90.

9 Lobban CD. The oxygen-conserving dive reflex re-examined as the principal contributory factor in the sudden infant death. Med Hypotheses 1995;44:273-7.

10 Lavezzi AM, Ottaviani G, Rossi L, et al. Hypoplasia of the parabrachial/ Kölliker-Fuse complex in perinatal death. Biol Neonate 2004;86:92-7.

11 Matturri L, Ottaviani G, Lavezzi AM, et al. Early atherosclerotic lesions of the cardiac conduction system arteries in infants. Cardiovasc Pathol 2004; 13:276-81.

12 Lobban CD. The human dive reflex as a primary cause of SIDS. A review of the literature. Med J Aust 1991;155:561-3.

13 Lavezzi AM, Ottaviani G, Mauri M, et al. Hypoplasia of the arcuate nucleus and maternal smoking during pregnancy, in perinatal and infant sudden unexpected death. Neuropathology 2004;24:284-9.

14 Matturri L, Biondo B, Suàrez-Mier MP, et al. Brain stem lesions in the sudden infant death syndrome: variability in the hypoplasia of the arcuate nucleus. Acta Neuropathol 2002;194:12-20.

15 Taylor JA, Sanderson M. A reexamination of the risk factors for the sudden infant death syndrome. J Pediatr 1995;126:887-91.

16 Storm H, Nylander G, Saugtad OD. The amount of brainstem gliosis in sudden infant death syndrome (SIDS) victims correlates with maternal cigarette smoking during pregnancy. Acta Paediatr 1999;88:13-18.

17 Matturri L, Lavezzi AM, Ottaviani G, et al. Intimal pre-atherosclerotic thickening of the coronary arteries in human fetuses of smoker mothers. J Thromb Haemost 2003; 1:2234-8. 